\title{
Targeted Knock-in of Transgenes into the CHO Cell Genome Using CRISPR-mediated Integration Systems
}

\author{
Ryusei IWAO ${ }^{1}$, Yoshinori KAWABE ${ }^{1}$, Mai MURAKAMI ${ }^{2}$, Akira ITO $^{1}$, Masamichi KAMIHIRA ${ }^{1,2 *}$ \\ ${ }^{1}$ Department of Chemical Engineering, Faculty of Engineering, Kyushu University, 744 Motooka, Nishi-ku, Fukuoka, Fukuoka 819- \\ 0395, Japan \\ ${ }^{2}$ Graduate School of Systems Life Sciences, Kyushu University, 744 Motooka, Nishi-ku, Fukuoka, Fukuoka 819-0395, Japan
}

\begin{abstract}
Biopharmaceutical proteins are usually produced by culturing recombinant Chinese hamster ovary $(\mathrm{CHO})$ cells. High producer cell lines are screened from transfected cells with random integration of target genes. Since transgene expression is susceptible to the surrounding environment of the integrated genomic locus, producer cell lines should be selected from a large number of recombinant cells with heterogeneous transgene insertion. In contrast, targeted integration into a characterized genomic locus allows for predictable transgene expression and less clonal variability, and thus stable production of target proteins can be expected. Genome editing technology based on programmable nucleases has recently emerged as a versatile tool for precise editing of target locus in the cell genome. Here, we demonstrated targeted knock-in of transgenes into the hypoxanthine phosphoribosyltransferase (hprt) locus of CHO cells using CRISPR/Cas9 and CRISPR-mediated precise integration into target chromosome (PITCh) systems. We also generated knock-in CHO cells based on the homology-independent targeted integration (HITI) system. We evaluated the knock-in efficiency of transgenes into the hprt locus using these systems.
\end{abstract}

\section{Introduction}

Chinese hamster ovary $(\mathrm{CHO})$ cells have been widely used as a host for biopharmaceutical protein production because they can produce proteins with post-translational modifications such as glycosylation and proper folding (Fisher et al., 2015). High producer cells have been conventionally screened from stable pools with random integration of transgenes, generated using gene amplification methods such as dihydrofolate reductase (DHFR)/methotrexate (MTX) and glutamine synthetase $(G S) /$ methionine sulfoximine (MSX) systems. The stable pool cells with random integration of transgenes exhibit diverse and unpredictable phenotypes in transgene expression (Noh et al., 2013), because transgene expression is susceptible to the surrounding environment of the integrated genomic locus. Thus, tedious screening efforts are required to establish high-producer clones.

In recent years, genome editing tools using artificial engineered enzymes such as transcription activator-like effector nuclease (TALEN) and clustered regularly interspaced short palindromic repeats (CRISPR)/CRISPR-associated protein 9 (Cas9) RNAguided nucleases have been developed for specific modification of target genes on the genome (Kim and Kim, 2014). These methods cause generation of DNA doublestrand breaks (DSBs) at desired sites on the genome, and gene knock-out and knock-in at the target site rely on the DSB repair mechanisms of cells, comprising two major pathways: non-homologous end joining (NHEL) and homologous recombination repair (HDR). In NHEJ, the target gene is disrupted by the frame shift caused by insertion and/or deletion at the target site. On the other hand, in HDR using a homologous sequence such as sister chromatid as a template, DSB is correctly repaired. Therefore, HDR-mediated knock-in of transgenes is possible by using a donor plasmid in which arm sequences homologous to the target sequence are added at the both ends of the target gene. Although HDR-mediated gene modification is frequently used for targeted knock-in of transgenes, the knock-in efficiency is generally known to be low.

Recently, as an efficient knock-in strategy, a precise integration into target chromosome (PITCh) system mediated by microhomology end joining (MMEJ) was reported (Nakade et al., 2014). Since the PITCh system does not depend on HDR like gene knock-in by NHEJ, it is expected to apply for cell types difficult to knock-in using conventional methods. In contrast, Suzuki et al. developed a simple knock-in procedure designated as homology-independent targeted integration (HITI) using non-homologous sequences in vivo (2016). This procedure exhibited higher knock-in efficiency compared with conventional genome editing methods using HDR, NHEJ, and PITCh methods. Figure 1 shows schematic drawings of these knock-in systems.

Targeted knock-in of a reporter gene using the HDRmediated CRISPR/Cas9 system has been reported in 2015 for $\mathrm{CHO}$ cells (Lee et al., 2015). Since then, there were some reports showing genomic modification (Lee et al.,

* Corresponding author: kamihira@chem-eng.kyushu-u.ac.jp 
2016) and transgene knock-in (Inniss et al., 2017) for $\mathrm{CHO}$ cells. In this study, we demonstrated targeted knockin of transgenes into the hypoxanthine phosphoribosyltransferase (hprt) locus of CHO cells, which is known to be beneficial for stable and high transgene expression (Wang et al., 2017), using CRISPR/Cas9 and CRISPR-mediated PITCh and HITI systems. We evaluated the transgene knock-in efficiency into the hprt locus using these systems.

\section{Materials and Methods}

\subsection{Plasmid construction}

Red fluorescence protein (DsRed) and $s c F v-F c$ genes derived from pIRES2-DsRed (Clontech, Palo Alto, USA) were inserted into the $\mathrm{pBA} / \mathrm{EF} 1 \alpha$ Pur vector (Takara Bio, Kusatsu, Japan) to generate donor plasmids for targeting the hprt locus. DNA fragments homologous to hprt exon 2 were amplified by PCR from CHO-K1 cell genome. The 5' and 3' $\mathrm{HR}$ regions were placed into upstream and downstream of marker gene expression cassettes (Psv40/Puro-PhEF1 $\alpha /$ DsRed), respectively, to prepare a donor vector for HR-mediated knock-in. To prepare donor vectors for the MMEJ-mediated PITCh system and for the NHEJ-mediated HITI system, DNA fragments corresponding to gRNAs for specific one PITCh-gRNA (v2) and hprtE2-gRNA of HITI were added to the vectors encoding transgene expression cassettes using PCR and standard molecular biology methods (pCRISv2/DsRed, pCRISv2/scFv-Fc and pHITI/DsRed).

As a Cas9 and sgRNA co-expression plasmid, pX330 (Addgene; Plasmid \#42230) was used. The sgRNA targeting exon 2 of the hprt locus was designed using the CRISPRdirect software (http://crispr.dbcls.jp). Two microhomologies- and PITCh-specific gRNA templates expression units under the control of human U6 promoter were inserted to generate all-in-one CRISPR/Cas9 vectors containing two gRNA expression units and a Cas9 cassette (pX330/E2-v2).

\subsection{Cells and medium}

CHO-K1 (Riken, Tsukuba, Japan) and recombinant CHO cells were cultured in a Ham's F12 basal medium (SigmaAldrich, St. Louis, USA) containing 10\% (v/v) fetal bovine serum (FBS) (BioWest, Nuaillé, France), 100 units/mL penicillin and $70 \mu \mathrm{g} / \mathrm{mL}$ streptomycin (Fujifilm Wako Pure Chemical Corporation, Osaka, Japan) in tissue-culture dishes or plates (Thermo Fisher Scientific, Waltham, MA, USA). Cells were incubated in a humidified $5 \%(\mathrm{v} / \mathrm{v}) \mathrm{CO}_{2}$ incubator at $37^{\circ} \mathrm{C}$.

\subsection{Transfection and cell screening}

CHO-K1 cells were seeded in wells of 24-well plates (Thermo Fisher Scientific) at a cell density of $1.2 \times 10^{5}$ cells/well. After $24 \mathrm{~h}$, the Cas9/sgRNA expression vector (pX330/E2) and donor vector were co-transfected using a lipofection reagent (Lipofectamine ${ }^{\circledR}$ 2000, Invitrogen).
Two days after transfection, $\mathrm{CHO}$ cells seeded at a cell density of $3.0 \times 10^{5}$ cells/well in 6 -well plates (Thermo Fisher Scientific) were cultured in F12 medium supplemented with $50 \mathrm{mg} / \mathrm{L}$ puromycin (Invitrogen) for 3 days. Five days after transfection, the cells were cultured for about 10 days in F12 medium supplemented with 50 $\mu \mathrm{M}$ 6-TG (Fujifilm Wako Pure Chemical Corporation, Osaka, Japan) and puromycin. The medium was replaced every three days during the culture period. Clones were isolated by the colony picking method. Picked clones were subjected to further experiments.

\subsection{Clone analysis}

Genomic DNA extracted from cell clones using a genomic DNA extraction kit (MagExtractor -genome-, Toyobo, Osaka, Japan) was subjected to genomic PCR analysis to assay targeted knock-in. Flow cytometry analysis (SH-800, Sony, Tokyo, Japan) was performed to analysis DsRed expression levels for the established clones.

\subsection{Genome editing efficiency}

The gRNA/Cas9 expression vectors were transfected into CHO-K1 cells using a lipofection reagent. Genomic DNA were extracted after 3 days of transfection. T7 endonuclease I (T7EI) assay were performed according to a previous report (Ran et al., 2013).

\section{Results}

\subsection{Design of guide RNAs for gene knock-in into the hprt locus of $\mathrm{CHO}$ cells}

First, the gRNAs for exons 2 and 3 of the hprt locus of $\mathrm{CHO}$ cells were designed and the corresponding gRNA expression vectors were constructed. Three days after transfection of the vectors into $\mathrm{CHO}-\mathrm{K} 1$ cells, genomic DNA was extracted from the cells. We evaluated the efficiency of genome editing using T7EI assay. The insertion/deletion (indel) mutation rate was measured as $15.1 \%$ for exon2 (Figure 2), but negligible for exon 3. Therefore, we used the gRNA for exon 2 for the further knock-in experiments.

\subsection{Targeted knock-in of transgenes into the hprt locus}

We evaluated the targeted knock-in efficiency of transgene cassettes among HR, CRIS-PITCh and HITI systems. CHO-K1 cells were transfected with each donor vector and corresponding Cas9-sgRNA co-expression plasmids. The amounts of Cas9-sgRNA and donor vectors used for transfection were each $400 \mathrm{ng} /$ well. The transfected cells seeded at the density of $3.0 \times 10^{5}$ cells/well in 6-well plates were selected using puromycin and followed by 6-TG treatment. After 10 day-culture for 
screening of transfectants, we counted the numbers of colonies showing resistance for both drugs (6-TG and puromycin) and DsRed expression. The colony numbers were 21, 28, and 141 for using HR, PITCh, and HITI vectors, respectively (Figure 3 ). The knock-in efficiency into the hprt locus using HITI system was 6.7-fold and 5.0-fold higher compared with those using HR and PITCh systems, respectively. In contrast, for the established clones, genomic PCR was performed using specific primer sets for 3' junction regions. When genomic PCR analysis for amplification around the 3 '-junction region was performed to evaluate targeted transgene knock-in for the clones established using each integration method, expected sequences for the amplicons were detected with $100 \%(5 / 5), 50 \%(4 / 8)$, and $17 \%(4 / 23)$ of clones using HR, PITCh, and HITI vectors, respectively.

A

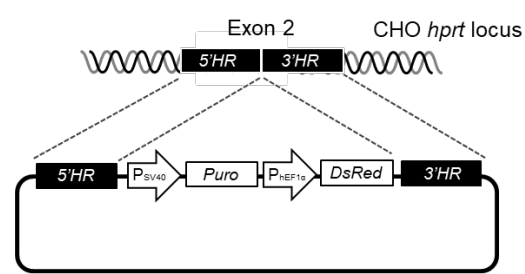

HR-mediated knock-in
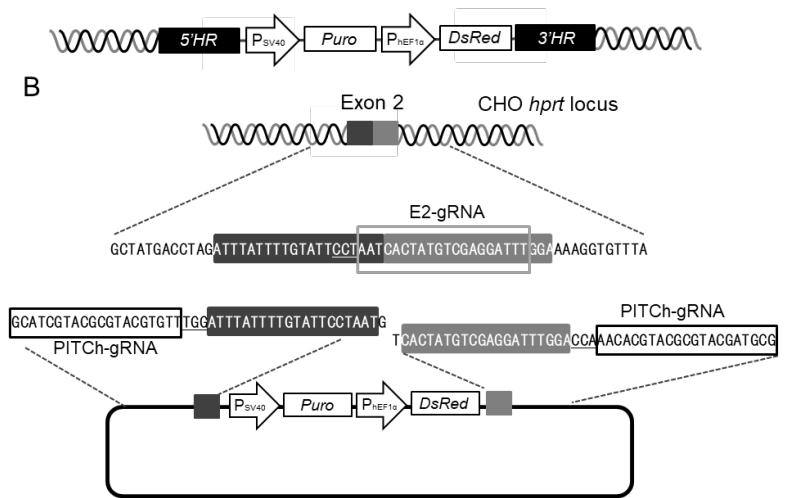

PITCh-mediated knock-in

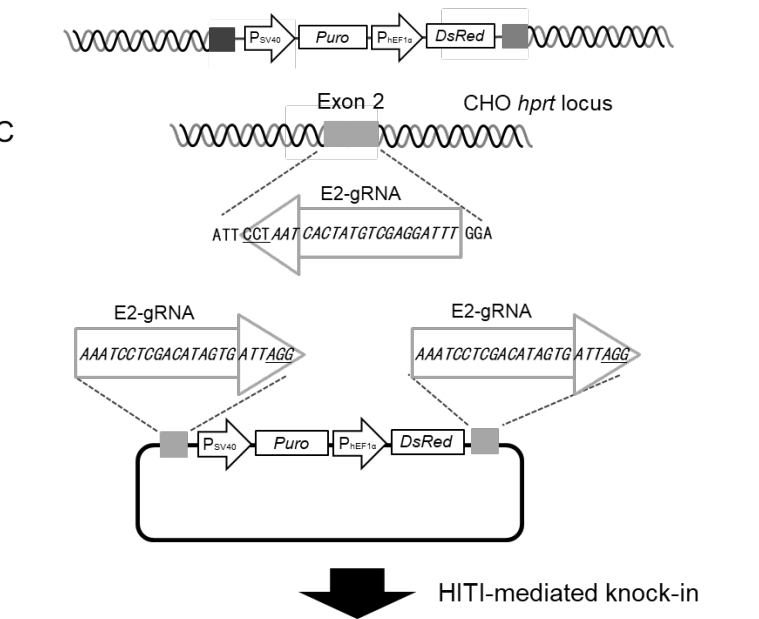

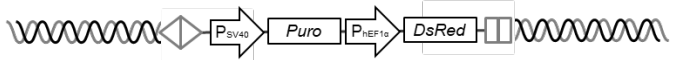

Figure 1. Schematic drawings of targeted knock-in using HR, PITCh and HITI systems.

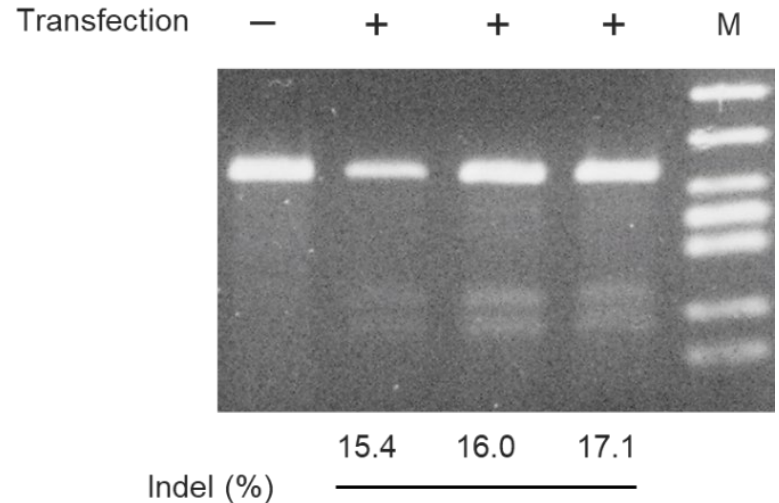

(ave) $16.1 \pm 0.70$

Figure 2. Genome editing efficiency for the hprt exon 2 of $\mathrm{CHO}$ cell genome.

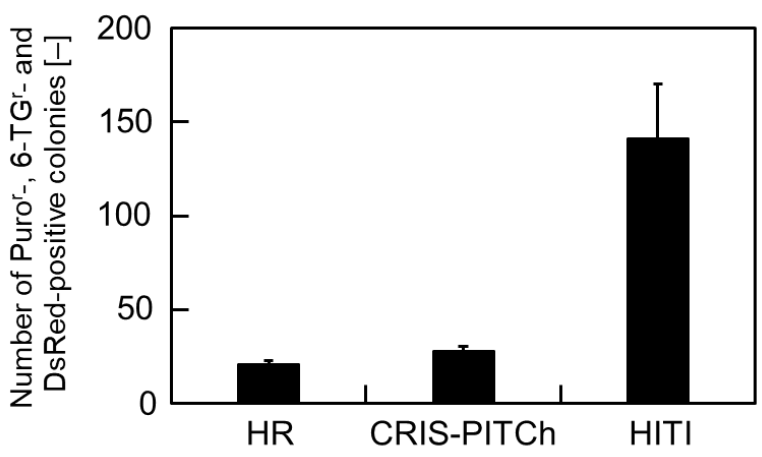

Figure 3. Colony formation.

\section{Discussion}

CHO cells are frequently used as host cells for the commercial production of biopharmaceutical proteins, including therapeutic antibodies. The biopharmaceutical protein production process using recombinant $\mathrm{CHO}$ cells has been well established and become a de facto standard in the pharmaceutical industry. In order to achieve high productivity, it is one of the most important steps to establish producer cells suitable for biomanufacturing. However, establishment of producer cells using conventional gene amplification procedures relies on unknown mechanisms. Therefore, stable and highly productive cell lines are screened from a large number of cells with a wide variety of transgene insertion sites. On the other hand, targeted integration into a characterized genomic locus is expected to obtain more predictable transgene expression levels for clones, and hence stable production of target proteins may be anticipated. In this study, we demonstrated targeted knock-in of transgenes into the hprt locus of CHO cells using CRISPR-mediated genome editing systems, and evaluated the transgene knock-in efficiency for these methods. 
In general, $\mathrm{CHO}$ cells have a feature of high frequency of transgene integration (random integration) as compared to other cell lines. Thus, a screening system that can distinguish between random and targeted integration of transgenes is very important for obtaining targeted knockin cells. Cells with knocked-out of $h p r t$ gene are selectable using a drug, 6-TG (Johnson, 2012). Therefore, in this study, double drug screening using puromycin and 6-TG effectively worked to establish knock-in cells, eliminating random and off-target integrants.

For the construction of PITCh- and HITI-mediated targeting vectors, short sequences for gRNA target (microhomology arms for PITCh and the same sequences as target locus for HITI) are easily added to donor vectors by PCR or insertion of synthetic oligonucleotides, simplifying donor vector construction compared with HR-mediated targeting vectors in which a target gene should be flanked with long homology arms (500-3,000 $\mathrm{bp})$. The colony forming efficiency exhibited 1.3 - and 6.7fold higher using PITCh- and HITI-mediated systems, respectively, compared with that using HR-mediated system, while targeted knock-in efficiency among the double drug-resistant clones was slightly lower for PITCh- and HITI-mediated systems. Recently, Aida et al. (2016) reported that overexpression of exonuclease $1 \mathrm{can}$ improve the PITCh-mediated system. The use of minicircle DNA vectors that lack bacterial backbone sequences enhanced the targeted knock-in efficiency in HITI-mediated system (Suzuki et al., 2016). In fact, we also showed that higher targeted integration efficiency in Cre/lox $P$-mediated site-specific integration was obtained using minicircle DNA as the donor vector (Wang et al., 2018). Furthermore, we have previously demonstrated that timing of drug addition and concentration of screening drugs greatly affected strict selection for the transgene integration into the hprt locus of CHO-K1 cells using the TALEN based-PITCh system (Sakuma et al., 2015). Thus, these technical improvements and optimization of screening conditions may further improve targeted knock-in efficiency.

In conclusion, we examined targeted knock-in of transgenes into the hprt locus of $\mathrm{CHO}$ cells using CRISPR-mediated integration systems. These systems effectively worked to generate targeted knock-in cells. The results indicate that the CRISPR-mediated targeted knock-in is a promising approach for $\mathrm{CHO}$ cell engineering.

\section{Acknowledgements}

This work was supported in part by developing key technologies for discovering and manufacturing pharmaceuticals used for next-generation treatment and diagnoses both from the Ministry of Economy, Trade and Industry, Japan (METI) and from Japan Agency for Medical Research and Development (AMED) under Grant Number JP17ae0101003.

\section{References}

Aida, T., S. Nakade, T. Sakuma, Y. Izu, A. Oishi, K. Mochida, H. Ishikubo, T. Usami, H. Aizawa, T. Yamamoto, and K. Tanaka; "Gene Cassette Knock-in in Mammalian Cells and Zygotes by Enhanced MMEJ," BMC Genomics, 17, 979 (2016)

Fischer, S., R. Handrick, and K. Otte; "The Art of CHO Cell Engineering: A Comprehensive Retrospect and Future Perspectives," Biotechnol. Adv., 33, 1878-1896 (2015)

Inniss, M. C., K. Bandara, B. Jusiak, T. K. Lu, R. Weiss, L. Wroblewska, and L. Zhang; "A Novel Bxb1 Integrase RMCE System for High Fidelity Site-specific Integration of mAb Expression Cassette in CHO Cells," Biotechnol. Bioeng., 114, 1837-1846 (2017)

Johnson, G. E.; "Mammalian Cell HPRT Gene Mutation Assay: Test Methods," Methods Mol. Biol., 817, 55-67 (2012)

Kim, H. and J. S. Kim; "A Guide to Genome Engineering with Programmable Nucleases," Nat. Rev. Genet., 15, 321-334 (2014)

Lee, J. S., T. B. Kallehauge, L. E. Pedersen, and H. F. Kildegaard; "Site-specific Integration in $\mathrm{CHO}$ Cells Mediated by CRISPR/Cas9 and Homology-directed DNA Repair Pathway," Sci. Rep., 5, 8572 (2015)

Lee, N., J. Shin, J. H. Park, G. M. Lee, S. Cho, and B. K. Cho; "Targeted Gene Deletion Using DNA-free RNAguided Cas9 Nuclease Accelerates Adaptation of $\mathrm{CHO}$ Cells to Suspension Culture," ACS Synth. Biol., 5, 1211$1219(2016)$

Nakade, S., T. Tsubota, Y. Sakane, S. Kume, N. Sakamoto, M. Obara, T. Daimon, H. Sezutsu, T. Yamamoto, T. Sakuma, and K. T. Suzuki; "Microhomology-mediated End-joining-dependent Integration of Donor DNA in Cells and Animals Using TALENs and CRISPR/Cas9," Nat. Commun., 5, 5560 (2014)

Noh, S. M., M. Sathyamurthy, and G. M. Lee; "Development of Recombinant Chinese Hamster Ovary Cell Lines for Therapeutic Protein Production," Curr. Opin. Chem. Eng., 2, 391-397 (2013)

Ran, F. A., P. D. Hsu, J. Wright, V. Agarwala, D. A. Scott, and F. Zhang; "Genome Engineering Using the CRISPRCas9 System,” Nat. Protoc., 8, 2281-2308 (2013)

Sakuma, T., M. Takenage, Y. Kawabe, T. Nakamura, M. Kamihira, and T. Yamamoto; "Homologous Recombination-Independent Large Gene Cassette Knockin in CHO Cells Using TALEN and MMEJ-Directed Donor Plasmids," Int. J. Mol. Sci., 16, 23849-23866 (2015) 
Suzuki, K., Y. Tsunekawa, R. Hernandez-Benitez, J. Wu, J. Zhu, E. J. Kim, F. Hatanaka, M. Yamamoto, T. Araoka, Z, Li, M. Kurita, T. Hishida, M. Li, E. Aizawa, S. Guo, S. Chen, A. Goebl, R. D Soligalla, J. Qu, T. Jiang, X. Fu, M. Jafari, C. R. Esteban, W. T. Berggren, J. Lajara, E. NuñezDelicado, P. Guillen, J. M. Campistol, F. Matsuzaki, G. H. Liu, P. Magistretti, K. Zhang, E. M. Callaway, K. Zhang, and J. C. Belmonte; "In vivo Genome Editing via CRISPR/Cas9 Mediated Homology-independent Targeted Integration," Nature, 540, 144-149 (2016)

Wang, X., Y. Kawabe, R. Kato, T. Hada, A. Ito, Y. Yamana, M. Kondo, and M. Kamihira; "Accumulative scFv-Fc Antibody Gene Integration into the hprt Chromosomal Locus of Chinese Hamster Ovary Cells," $J$. Biosci. Bioeng., 124, 583-590 (2017)

Wang, X., Y. Kawabe, T. Hada, A. Ito, and M. Kamihira; "Cre-Mediated Transgene Integration in Chinese Hamster Ovary Cells Using Minicircle DNA Vectors.," Biotechnol. J., 13, e1800063 (2018) 